\title{
Type II Parametric Downconversion in a Poled Fiber
}

\author{
E. Y. Zhu, ${ }^{1, *}$ E. A. Lee-Kim Koon, ${ }^{1}$ L. Qian, ${ }^{1}$ L. G. Helt, ${ }^{2}$ M. Liscidini, ${ }^{2,3}$ J. E. Sipe, ${ }^{2}$ \\ C. Corbari, ${ }^{4}$ A. Canagasabey, ${ }^{4,5}$ M. Ibsen, ${ }^{4}$ P. G. Kazansky, \\ ${ }^{1}$ Dept. of Electrical and Computer Engineering, University of Toronto, 10 King's College Rd., Toronto, ON M5S 3G4, Canada \\ ${ }^{2}$ Dept. of Physics, University of Toronto, 60 St. George St., Toronto, ON M5S 1A7, Canada \\ ${ }^{3}$ Current address: Dipartimento di Fisica A. Volta, Universita degli Studi di Pavia, Via Bassi 6 I-27100, Italy \\ ${ }^{4}$ Optoelectronics Research Centre, University of Southampton, SO17 1BJ, United Kingdom \\ ${ }^{5}$ Current address: School of Physics, University of Sydney, New South Wales 2006, Australia \\ *Corresponding author: eric.zhu@utoronto.ca
}

Abstract: We report photon-pair generation at the $1.5-\mu \mathrm{m}$ telecom band via continuous-wave type-II parametric downconversion in a birefringent periodically-poled silica fiber. The time- and polarization-correlations of the downconverted light are examined.

(C) 2011 Optical Society of America

OCIS codes: (190.4370) Nonlinear optics, fibers; (270.0270) Quantum optics

\section{Introduction}

Single photon and correlated photon-pair sources are required for many applications in quantum information. An experimental realization of correlated photon pair generation can be found through the nonlinear optical process of spontaneous parametric down-conversion (SPDC). Conventional optical fiber composed of fused silica, however, does not possess the second-order optical nonlinearity $\left(\chi^{(2)}\right)$ required for SPDC. It is still possible to generate photon pairs in fiber with spontaneous four-wave-mixing, a third-order process, but the presence of spontaneous Raman scattering requires that the signal and idler be greatly separated in wavelength [1] or that the fiber be cryogenically cooled [2]. Such problems can be alleviated if the second-order nonlinearity were present in fused silica.

One can induce a non-zero $\chi^{(2)}$ in optical fiber through thermal poling [3]. The process is performed with specialty twin-hole fiber (a cross-section of which is shown in Fig. 1a inset), in which two large air-holes sandwich the core. The induced $\chi^{(2)}$ arises from a frozen-in DC field $E_{x}^{D C}$ - present in the core region of the fiber after poling - and the Kerr nonlinearity: $\chi_{i j k}^{(2)}=3 \chi_{i j k x}^{(3)} E_{x}^{D C}$. The DC field is taken by convention to be in the $x$ direction. This model gives rise to the following relation between the $\chi^{(2)}$ tensor elements, which have been found to be experimentally valid for our fiber [4]: $\chi_{x x x}^{(2)}=3 \chi_{x y y}^{(2)}=3 \chi_{y x y}^{(2)}=3 \chi_{y y x}^{(2)}$.

Our poled fiber is quasi-phase-matched (or periodically-poled) [3] for the second-harmonic generation (SHG) of $\lambda_{S H} \approx 775 \mathrm{~nm}$ light in the $\mathrm{LP}_{01}$ mode. The four non-zero elements of the $\chi^{(2)}$ tensor result in the presence of three distinct SHG signals $(y+y \rightarrow x, x+x \rightarrow x, y+x \rightarrow y)$ (Fig. 1a). The fiber birefringence, which we attribute to the fiber geometry (Fig. 1a inset), allows for the spectral separation of the three phase-matchings.

From Fig. 1a, we find that the type-II SPDC phase-matching $(y \rightarrow x+y)$ is perfectly quasi-phase-matched when $y$ polarized CW pump light of wavelength $\lambda_{P} \approx 774.7 \mathrm{~nm}$ is launched into the fiber. At this pump wavelength, however, the signal and idler are degenerate (Fig. 1b); by detuning $\lambda_{P}$ to $774.9 \mathrm{~nm}$, it is possible to deterministically separate the signal and idler in wavelength using a C- and L-Band wavelength-division multiplexer (WDM).

In this work, we observe the time- and polarization-correlated nature of the downconverted type-II photon pairs in a 20-cm long periodically-poled silica fiber (PPSF). Previous work [5] utilized lower-efficiency poled fibers with poorer coincidence-to-accidental ratios, where fiber birefringence was (presumably) not present to allow for the observation of polarization correlation.

\section{Experiment}

A Ti:Sapph laser (Fig. 1c) is used as the CW pump, with its wavelength $\lambda_{P}$ tuned to $774.88 \pm 0.05 \mathrm{~nm}$. The pump polarization is adjusted with a polarizer (Pol. P), quarter-, and half-waveplates (QWP, HWP) before being launched into an objective lens (OBJ) and coupled into a single-mode telecom fiber (SMF-28) pigtail that is fusion-spliced to the PPSF. The pump power within the PPSF is approximately $50 \mathrm{~mW}$ (not all of which is in the $\mathrm{LP}_{01}$ mode, as the fiber is multi-mode at $\lambda_{P}$ ). At the output end of the PPSF, the downconverted light is filtered out from the 775-nm pump using three cascaded 1550/775 nm WDMs, allowing for greater than $110 \mathrm{~dB}$ pump suppression. The pump power and wavelength are monitored with a power meter and optical spectrum analyzer (respectively). The signal and idler are then deterministically split in wavelength using a C-/L-Band WDM. The total insertion loss at $1.5-\mu \mathrm{m}$ for the system is $6.5 \mathrm{~dB}$ per leg, and quantum efficiencies of the single-photon detectors (SPDs) are $10 \%$ (C-Band) and $6 \%$ (L-Band). On the C-Band leg, a fiber-polarization controller (FPC1) and fiber-based polarizer (Pol. 1) ensure that only $x$-polarized $\left(\theta_{1}=0\right)$ light enters the SPD. On the L-Band leg, FPC2 and a free-space polarizer $\left(\theta_{2}\right)$ allow us to vary the polarization monitored, from $x$ to $y$ and all other linear polarizations in between; it is initially set to $y\left(\theta_{2}=90^{0}\right)$. 

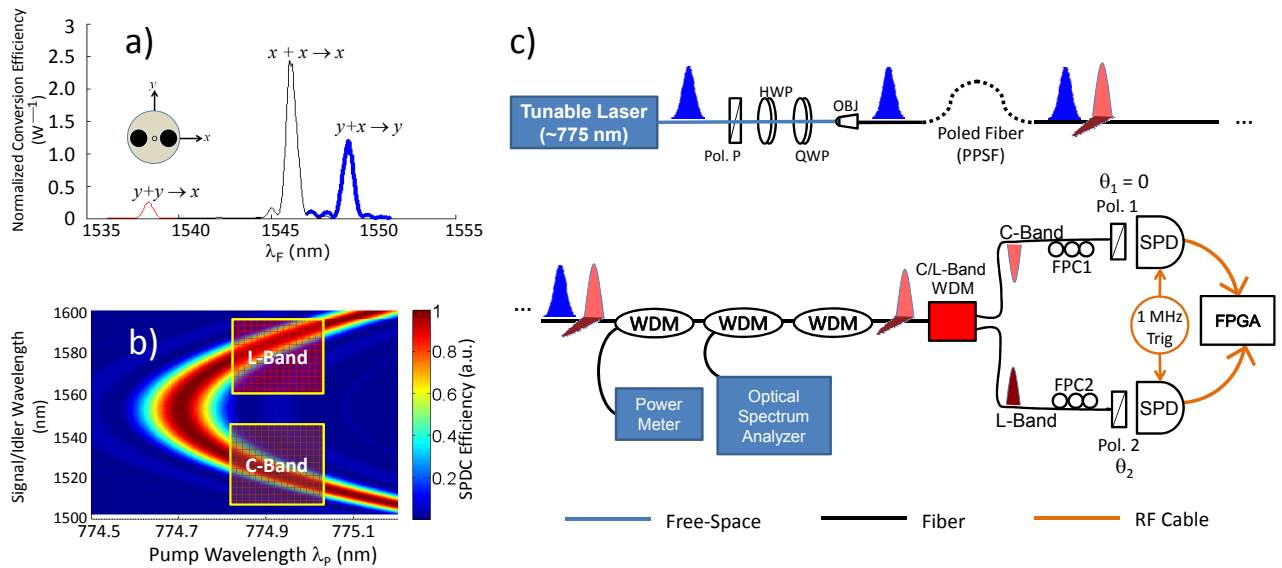

Free-Space $\longrightarrow$ Fiber Cable

Fig. 1. a) Experimental SHG spectrum for our poled fiber. The inset shows the fiber cross-section of the poled fiber; note that the DC-field $E_{x}^{(D C)}$ is aligned to one of the fiber's principal axes $(x)$. b) Theoretical SPDC tuning curve for the type-II phase-matching; detuning $\lambda_{P}$ to the red results in spectral separation of the downconverted light. c) Experimental setup for measuring the time- and polarization-correlations of the photon pairs; the blue 'pulse' represents the pump light (which is actually $\mathrm{CW}$ ), and the red/magenta pulses represent the downconverted signal and idler.

The coincidence measurement involves triggering both the SPDs synchronously at $f=1 \mathrm{MHz}$. A relative electronic delay between the trigger signals is implemented for the time-correlation measurement. Due to the narrow optical gates (400 ps) of the SPDs, the relative delay resolution must be small (100 ps). A field-programmable gate array (FPGA) is used as coincidence counter; a coincidence is counted when the SPDs both fire within $50 \mathrm{~ns}$ of each other. As the downconverted signal and idler photons are generated simultaneously, the arrival times at their respective SPDs should be correlated, and the coincidence counts should peak for a specific relative delay. Here (Fig. 2a), we find that the relative delay is $10.1 \mathrm{~ns}$, and that the coincidence-to-accidental ratio (CAR) is on the order of 30:1 (when the C-Band leg is $x$-polarized, and L-Band leg $y$-polarized). The integration time for each delay value is 200 seconds.

For polarization-correlation, the polarizer angle $\left(\theta_{2}\right)$ on the L-Band leg (Fig. 1c) is allowed to vary from $0^{\circ}$ to $180^{\circ}$, and coincidences are measured for various angles. The C-Band leg, meanwhile, is fixed to the $x$-polarization. The relative trigger delay is set to $10.1 \mathrm{~ns}$ to optimize coincidence counts, and an integration time of $200 \mathrm{~s}$ is used for each value of $\theta_{2}$. We observe clearly (Fig. 2b) that the coincidence counts are maximized when the L-Band leg is $y$-polarized $\left(\theta_{2} \approx 90^{\circ}\right)$, and minimized when it is $x$-polarized $\left(\theta_{2} \approx 0^{\circ}, 180^{\circ}\right)$. These results are consistent with the type-II SPDC phase-matching $y \rightarrow x+y$.
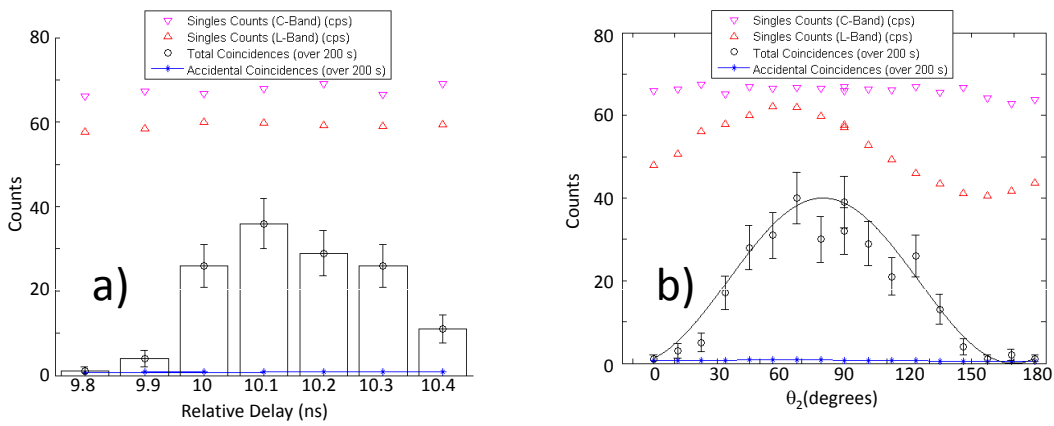

Fig. 2. a) Results of the time-correlation experiment show that the coincidence-to-accidental ratio (CAR) exceeds 30:1, and that coincidences are maximized when the relative trigger delay is $10.1 \mathrm{~ns}$. The $\mathrm{C}$-Band polarizer is set to $x$, while the L-Band is $y$-polarized. b) Polarization-correlation measurements show that when the C-Band leg is $x$-polarized $\left(\theta_{1}=0^{0}\right)$, the coincidence measurements are maximized when the L-Band leg is $y$-polarized $\left(\theta_{2} \approx 90^{\circ}\right)$, as expected for type-II downconversion. Singles counts are measured in counts per second (cps), and the accidental coincidences $(A c c)$ are calculated using the singles counts $\left(s_{C}, s_{L}\right)$ and trigger rate $f(=1 \mathrm{MHz})$ of the SPDs: $A c c=s_{C} \times s_{L} / f$.

\section{Conclusion}

We have demonstrated time- and polarization-correlations in the SPDC pairs produced by type-II quasi-phase-matching in a birefringent PPSF. Future work will include observation of polarization entanglement from the source.

\section{References}

1. J. Rarity, J. Fulconis, et al., Opt. Express 13, 534-544 (2005).

2. X. Li, P. L. Voss, et al., Phys. Rev. Lett. 94, 053601 (2005).

3. A. Canagasabey, C. Corbari, et al., Opt. Lett. 32, 1863-1865 (2007).

4. E. Y. Zhu, L. Qian, et al., Opt. Lett. 35, 1530-1532 (2010).

5. K. P. Huy, A. T. Nguyen, et al., Opt. Express 15, 4419-4426 (2007). 